\title{
Semilocal and Hybrid Density Embedding Calculations of Ground-State Charge-Transfer Complexes
}

\author{
S. Laricchia, ${ }^{1}$ E. Fabiano, ${ }^{2}$ and F. Della Sala ${ }^{2,1}$ \\ ${ }^{1}$ Center for Biomolecular Nanotechnologies @UNILE, \\ Istituto Italiano di Tecnologia (IIT), Via Barsanti, 73010 Arnesano (LE), Italy \\ ${ }^{2}$ National Nanotechnology Laboratory (NNL), Istituto Nanoscienze-CNR, Via per Arnesano 16, 73100 Lecce, Italy
}

(Dated: September 1, 2018)

\begin{abstract}
We apply the frozen density embedding method, using a full relaxation of embedded densities through a freeze-and-thaw procedure, to study the electronic structure of several benchmark groundstate charge-transfer complexes, in order to assess the merits and limitations of the approach for this class of systems. The calculations are performed using both semilocal and hybrid exchangecorrelation (XC) functionals. The results show that embedding calculations using semilocal XC functionals yield rather large deviations with respect to the corresponding supermolecular calculations. Due to a large error cancellation effect, however, they can often provide a relatively good description of the electronic structure of charge-transfer complexes, in contrast to supermolecular calculations performed at the same level of theory. On the contrary, when hybrid XC functionals are employed, both embedding and supermolecular calculations agree very well with each other and with the reference benchmark results.

In conclusion, for the study of ground-state charge-transfer complexes via embedding calculations hybrid $\mathrm{XC}$ functionals are the method of choice due to their higher reliability and superior performance.
\end{abstract}

PACS numbers:

\section{INTRODUCTION}

In the last years a considerable interest in densityembedding methods has led to a widespread development of subsystem approaches [1 21] within density functional theory (DFT) 22, 23]. In particular, the frozen density embedding (FDE) method 24 36] has emerged as a popular tool to treat intermolecular interactions in a simple and potentially exact embedding scheme.

In the FDE approach the total electronic density of a system is partitioned into a set of subsystem densities (e.g., for two subsystems A and B: $\rho=\rho_{\mathrm{A}}+\rho_{\mathrm{B}}$ ). The total electronic energy is then written as

$$
\begin{aligned}
& E[\rho]=E\left[\rho_{\mathrm{A}}+\rho_{\mathrm{B}}\right]=T_{\mathrm{s}}\left[\rho_{\mathrm{A}}\right]+T_{\mathrm{s}}\left[\rho_{\mathrm{B}}\right]+T_{\mathrm{s}}^{\mathrm{nadd}}\left[\rho_{\mathrm{A}}, \rho_{\mathrm{B}}\right]+ \\
& +\frac{1}{2} \iint \frac{\left(\rho_{\mathrm{A}}\left(\mathbf{r}_{1}\right)+\rho_{\mathrm{B}}\left(\mathbf{r}_{1}\right)\right)\left(\rho_{\mathrm{A}}\left(\mathbf{r}_{2}\right)+\rho_{\mathrm{B}}\left(\mathbf{r}_{2}\right)\right)}{\left|\mathbf{r}_{1}-\mathbf{r}_{2}\right|} d \mathbf{r}_{1} d \mathbf{r}_{2}+ \\
& +\int\left(v_{\mathrm{ext}}^{\mathrm{A}}\left(\mathbf{r}_{1}\right)+v_{\mathrm{ext}}^{\mathrm{B}}\left(\mathbf{r}_{1}\right)\right)\left(\rho_{\mathrm{A}}\left(\mathbf{r}_{1}\right)+\rho_{\mathrm{B}}\left(\mathbf{r}_{1}\right)\right) d \mathbf{r}_{1}+ \\
& +E_{\mathrm{xc}}\left[\rho_{\mathrm{A}}\right]+E_{\mathrm{xc}}\left[\rho_{\mathrm{B}}\right]+E_{\mathrm{xc}}^{\mathrm{nadd}}\left[\rho_{\mathrm{A}}, \rho_{\mathrm{B}}\right],
\end{aligned}
$$

where $T_{\mathrm{s}}$ is the noninteracting kinetic energy functional, the external potential was partitioned as $v_{\text {ext }}=v_{\text {ext }}^{\mathrm{A}}+$ $v_{\text {ext }}^{\mathrm{B}}$, and the superscript nadd denotes the nonadditive energy contributions to the kinetic and exchangecorrelation (XC) energies. The minimization of the energy of Eq. (11) with respect to one of the subsystem electron densities (e.g., $\rho_{\mathrm{A}}$ ), keeping the other one fixed and using the expansion of the electron density in terms of (auxiliary) Kohn-Sham orbitals $\psi_{i}^{\mathrm{A}}$, leads to the Kohn-Sham equations with constrained electron density
(KSCED) 24, 25]

$$
\left(-\frac{1}{2} \nabla^{2}+v^{\mathrm{KS}}\left[\rho_{\mathrm{A}}\right](\mathbf{r})+v_{\mathrm{emb}}\left[\rho_{\mathrm{A}} ; \rho_{\mathrm{B}}\right](\mathbf{r})\right) \psi_{i}^{\mathrm{A}}=\varepsilon_{i}^{\mathrm{A}} \psi_{i}^{\mathrm{A}}
$$

with

$$
\begin{aligned}
v^{\mathrm{KS}}\left[\rho_{\mathrm{A}}\right](\mathbf{r}) & =\int \frac{\rho_{\mathrm{A}}\left(\mathbf{r}_{1}\right)}{\left|\mathbf{r}-\mathbf{r}_{1}\right|} d \mathbf{r}_{1}+v_{\mathrm{ext}}^{\mathrm{A}}(\mathbf{r})+\frac{\delta E_{\mathrm{xc}}\left[\rho_{\mathrm{A}}\right]}{\delta \rho_{\mathrm{A}}(\mathbf{r})} \\
v_{\mathrm{emb}}\left[\rho_{\mathrm{A}} ; \rho_{\mathrm{B}}\right](\mathbf{r}) & =v_{\mathrm{ext}}^{\mathrm{B}}(\mathbf{r})+\int \frac{\rho_{\mathrm{B}}\left(\mathbf{r}_{1}\right)}{\left|\mathbf{r}-\mathbf{r}_{1}\right|} d \mathbf{r}_{1}+ \\
& +\frac{\delta T_{\mathrm{s}}^{\mathrm{nadd}}\left[\rho_{\mathrm{A}}, \rho_{\mathrm{B}}\right]}{\delta \rho_{\mathrm{A}}(\mathbf{r})}+\frac{\delta E_{\mathrm{xc}}^{\mathrm{nadd}}\left[\rho_{\mathrm{A}}, \rho_{\mathrm{B}}\right]}{\delta \rho_{\mathrm{A}}(\mathbf{r})}(4)
\end{aligned}
$$

The KSCED of Eq. (2) provide the solution for the ground state of subsystem A subject to the interaction with the frozen density of the subsystem B. If the KSCED of both subsystems are considered in a freezeand-thaw procedure [25, 37], the full variational solution for the total system, equivalent to the usual KohnSham solution, is obtained, except for approximations eventually included in the nonadditive interaction terms. Henceforth, the acronym FDE wil be used to refer to this approach.

So far, numerous applications of the FDE-based method, using the KSCED approach, have been presented in literature to study the interaction of noncovalently bound (or weakly interacting) molecular complexes or solvated molecules [26 34]. In this cases in fact the nonadditive kinetic energy term can be efficiently approximated by means of semilocal approximations [3841]. However, because in the conventional formulation 
the use of KSCED is limited to local/semilocal XC functionals, most studies have focused on hydrogen-bond and dipole-dipole systems, since these interactions are dominated by electrostatic and polarization effects that can be described relatively well at the semilocal level of theory [42, 43].

Recently the method has been also extended to the generalized Kohn-Sham (GKS) framework [44, 45], so that hybrid functionals can be considered as well. In this approach, named GKS-FDE, the nonadditive kinetic and $\mathrm{XC}$ terms in Eq. (4) are replaced by the nonadditive direct and residual interactions $F_{\mathrm{s}}^{\text {nadd }}$ and $R_{\mathrm{s}}^{\text {nadd }}$ which include respectively the orbital-dependent and the explicitly density-dependent kinetic and XC terms. The presence of nonlocal and/or orbital-dependent terms makes however the direct implementation of the method rather involved. Therefore, a practical computational scheme was proposed where these terms are approximated with local functionals [44]. Thus, in practice in the GKS-FDE method the electronic structure is evaluated using a nonlocal exchange operator for the intra-subsystem XC effects but with an embedding potential evaluated at the local/semilocal level of theory. A similar approximation was also proposed for the extention of the KSCED to the use of orbital-dependent optimized effective potentials [46].

The GKS-FDE method was tested for a variety of hydrogen bond and dipole-dipole interactions and proved to be a very good approach for embedded simulations with hybrid functionals 44 46. In particular, with respect to FDE-based calculations employing semilocal XC functionals, it enables a general reduction of the embedding errors, because of the smaller overlap of subsystem densities at the hybrid level, and it allows a better description of subsystem properties due to the removal of most of the self-interaction error (SIE) [46 48]. Furthermore, it makes it possible to extend the application of FDE-based techniques to treat systems that are typically poorly described by semilocal XC functionals.

An example of the latter is given by ground-state charge-transfer complexes (also called donor-acceptor complexes). These systems derive in fact a considerable portion of their stabilization energy from an electron charge-transfer between two centers. Thus, unlike for hydrogen bonds or dipole-dipole interactions, where electrostatic and polarization effects dominate, the interaction in charge-transfer complexes is largely determined by orbital interactions. As a consequence, the description of ground-state charge-transfer complexes is very challenging for conventional semilocal XC functionals because of the SIE inherent in these methods. On the other hand, a good performance is generally obtained for hybrid functionals, especially when rather large portions of HartreeFock exchange are included 4965$]$. Similar results also concern the charge transfer between organic molecules and metal substrates [66, 67].
Due to the difficult application of DFT-based methods using semilocal XC functionals to these systems, in the last years only few FDE investigations treated ground-state charge-transfer complexes and related problems [36, 41, 68 70]. Some of them [41, 70] made use of complicated or ad hoc reparametrized kinetic energy functionals in order to obtain improved kinetic embedding potentials and improve the description of the system. However, these kinetic functionals cannot be routinely applied in FDE calculations for large systems. On the other hand, an extensive investigation of different kinetic energy functionals [68] showed that most popular kinetic energy approximations perform similarly and reasonably well for complexes displaying small/moderate charge transfer, so that it appears reasonable to consider in such applications the same kinetic energy approximation as those commonly used for hydrogen bond and dipole-dipole interactions. The role of the $\mathrm{XC}$ functionals in embedding calculations dealing with chargetransfer systems, instead, was not given much attention so far [36].

In this paper we cover this issue and demonstrate how hybrid functionals can be practically utilized within an embedding scheme to obtain an improvement of the performance in the description of embedded systems for a benchmark of charge-transfer complexes.

\section{METHOD AND COMPUTATIONAL DETAILS}

The KSCED [24, 25] and GKS-FDE [4, 45] approaches are implemented in the TURBOMOLE program package [71], version 6.4. All calculations were performed using the FDE script, which uses a freeze-andthaw procedure [37] to guarantee the full relaxation of the embedded ground-state electron density of both subsystems, until dipole moments of the embedded subsystems converged to $10^{-3}$ au. A supermolecular def2-TZVPPD 72, 73] basis set was employed in all calculations to expand the subsystem electron densities. A monomolecular (or too small) basis set is in fact insufficient to carry on FDE calculations with good accuracy, resulting in basisset errors larger than the FDE errors and the differences between the methods. The def2-TZVPPD basis set adds diffuse basis functions to the def2-TZVPP [73] basis set, thus granting an accurate representation for the electron densities even at the relatively large bonding distances characteristic of the systems under consideration. Very accurate integration grids were employed to minimize numerical errors. Additional details about our implementation and computational procedure are reported in Refs. 44 - 46.

In our investigation we considered a set of twelve representative ground-state charge-transfer complexes $\left(\mathrm{C}_{2} \mathrm{H}_{4}\right.$ $\mathrm{F}_{2}, \mathrm{NH}_{3}-\mathrm{F}_{2}, \mathrm{C}_{2} \mathrm{H}_{2}-\mathrm{ClF}, \mathrm{HCN}-\mathrm{ClF}, \mathrm{NH}_{3}-\mathrm{Cl}_{2}, \mathrm{H}_{2} \mathrm{O}-\mathrm{ClF}$, $\mathrm{NH}_{3}-\mathrm{ClF}, \mathrm{HCN}-\mathrm{NF}_{3}, \mathrm{HNC}-\mathrm{NF}_{3}, \mathrm{HF}-\mathrm{NF}_{3}, \mathrm{ClF}-\mathrm{NF}_{3}$, and 
$\mathrm{C}_{2} \mathrm{H}_{4}-\mathrm{Cl}_{2}$ ). The geometries and the reference binding energies were taken from Refs. 49, 74, except for the $\mathrm{C}_{2} \mathrm{H}_{4}$ $\mathrm{Cl}_{2}$ complex (for this latter case we optimized the geometry at the MP2/aug-cc-pVTZ level of theory 75-78] and calculated the reference binding energy, in analogy with Ref. 79] as $E_{b}^{r e f}=E_{b}^{\mathrm{CCSD}(\mathrm{T})}+\Delta E_{b}^{\mathrm{MP} 2}$, where $E_{b}^{\mathrm{CCSD}(\mathrm{T})}$ is the binding energy computed at the $\operatorname{CCSD}(\mathrm{T}) /$ aug-ccpVQZ level of theory [76 78, 80] and $\Delta E_{b}^{\mathrm{MP} 2}$ is the difference between the MP2 binding energies computed at the complete basis set limit (56-extrapolation [81]) and with an aug-cc-pVQZ basis set; the resulting binding energy is in very good agreement to that reported in Ref. 51).

The calculations were performed using conventional semilocal XC functionals (BLYP 82, 83], PBE 84]) as well as hybrid methods (PBE0 [85] and BHLYP [82, 83]). All calculations were performed using DFT-D3 dispersion corrections 86 . To compute the embedding nonadditive interactions, the nonadditive noninteracting kinetic energy term was approximated using the GGA functional revAPBEk [39, 40]. In the case of hybrid XC functionals also the nonadditive exchange term was approximated: the PBE and the B88x [82] exchange functionals were employed for PBE0 and BHYLP, respectively; the resulting methods are indicated as PBE0/PBE and $\mathrm{BH}-$ LYP/BLYP. In the case of semilocal XC functionals instead, no approximation for the nonadditive exchange contribution to the embedding potential is required: the same exchange functional is used for the embedding potential, subsystem and conventional supermolecule KS calculations.

The quality of embedded densities was investigated by considering the absolute deviation of plane-averaged embedding density, defined as

$\Delta \bar{\rho}(z)=\int\left|\rho_{\mathrm{A}}(x, y, z)+\rho_{\mathrm{B}}(x, y, z)-\rho^{\mathrm{GKS}}(x, y, z)\right| d x d y$

where $\rho^{\mathrm{GKS}}$ is the electron density obtained from a (generalized) KS calculation on the whole system. A quantitative measurement of the absolute error associated with a given embedded density was then obtained by computing the embedding density error

$$
\xi=\frac{1000}{N} \int \Delta \bar{\rho}(z) d z
$$

with $N$ being the number of electrons. In the evaluation of $\xi$ only valence densities were considered to avoid numerical problems related to the high value assumed by the electron density in the core regions and in consideration of the fact that the core density is not important for the determination of chemical and physical properties of interaction between the subsystems, which are of interest here.

The net charge on the $i$-th subsystem (monomer charge) was calculated for each method by considering a grid-based atoms-in-the-molecule partitioning [87] of the electron density into atomic basins $\Omega_{j}$ as

$$
\nu_{i}=\sum_{j=1}^{N_{i}} \int_{\Omega_{j}} \rho(\mathbf{r}) d \mathbf{r}-Z_{j}
$$

with $Z_{j}$ the nuclear charge of the $j$-th atom and $N_{i}$ the number of atoms in the $i$-th subsystem. For two interacting subsystems $\nu_{\mathrm{A}}+\nu_{\mathrm{B}}=0$ and thus we define the charge transfer as $\chi=\left|\nu_{\mathrm{A}}\right|=\left|\nu_{\mathrm{B}}\right|$. Reference values for the charge transfer were evaluated using relaxed MP2 densities computed with an aug-cc-pVTZ basis set.

Finally, to inspect the quality of the embedding energies we considered the error defined as

$$
\Delta E\left[\rho_{\mathrm{A}}, \rho_{\mathrm{B}}, \rho^{\mathrm{GKS}}\right]=E^{\mathrm{emb}}\left[\rho_{\mathrm{A}}, \rho_{\mathrm{B}}\right]-E^{\mathrm{GKS}}\left[\rho^{\mathrm{GKS}}\right],
$$

where $E^{\mathrm{emb}}$ is the embedding energy, computed using the embedding densities into Eq. (10), and $E^{\mathrm{GKS}}$ is the energy resulting from a conventional (generalized) KS calculation on the full system. Further analysis was also performed considering, according to Ref. 45, the error decomposition

$$
\begin{aligned}
\Delta E\left[\rho_{A}, \rho_{B}, \rho^{\mathrm{GKS}}\right]= & \Delta T\left[\rho_{A}, \rho_{B}, \rho^{\mathrm{GKS}}\right]+\Delta D\left[\rho, \rho^{\mathrm{GKS}}\right]+ \\
& +\Delta X^{S}\left[\rho_{A}, \rho_{B}, \rho^{\mathrm{GKS}}\right],
\end{aligned}
$$

where the error on embedding energy is partitioned into kinetic $(\Delta T)$, exchange $\left(\Delta X^{S}\right)$, and relaxation $(\Delta D)$ contributions. For full details see Ref. 45 .

\section{RESULTS AND DISCUSSION}

In this section we report and analyze the results of the FDE-based calculations on the charge-transfer complexes. We evaluate the performance of hybrid and semilocal XC functionals (BHLYP, PBE0, BLYP, and $\mathrm{PBE})$, elucidating what are the roles of the exact exchange, of the geometry and of the charge transfer between the subsystems.

\section{Embedding energy}

In Tab. \we report the binding energies obtained from different embedding methods $\left(E_{b}^{\mathrm{emb}}\right)$ and the corresponding results from conventional DFT calculations on the whole supermolecular system $\left(E_{b}^{\mathrm{GKS}}\right)$. In addition, we report the deviation of these results from accurate reference values ( $\Delta^{\mathrm{emb}}$ and $\Delta^{\mathrm{GKS}}$, respectively) and the error on embedding energies as defined in Eq. (8). We note that the embedding error on the total energy is exactly equivalent to the difference of the binding energies computed respectively from embedding and conventional supermolecular calculations (i.e. the DFT calculation 
on the whole system), since the contributions from isolated subsystems exactly cancel out. Moreover, all binding energies reported in Tab. I are dispersion-corrected and, because we use a supermolecular basis set, the basis set superposition error for the total-system calculation is treated in the same way in both DFT and FDE-based methods (Boys-Bernardi correction [88]). In Tab. [ we also report in the last lines the mean signed error (MSE), the mean absolute error (MAE), and the mean absolute relative error (MARE) with respect to the reference binding energies.

A first inspection of the results, focusing the attention on $E_{b}^{\mathrm{GKS}}$ and $\Delta^{\mathrm{GKS}}$, confirms the finding, well established in literature [49, 51, 53, 54, 56, 89], that hybrid functionals outperform semilocal ones for the calculation of interaction energies of ground-state chargetransfer complexes. In fact, BHLYP with a MARE of $16.63 \%$ is the most accurate method. However, when interaction energies from embedding calculations are considered (i.e. $E_{b}^{\mathrm{emb}}$ ), all methods yield rather similar performances with MAREs in the range 30-37\% (for a discussion of the issues related to the comparison of embedding binding energies with experimental or theoretical references see e.g. Refs. [26, 27, 90]). This suggests that a strong error cancellation occurs for embedding calculations using semilocal functionals, while the cancellation is much less in the case of hybrid approaches. The reason for this behavior can be traced back to the fact that, in general, conventional semilocal functionals overestimate significantly the binding energy of charge-transfer complexes [42, 49, 51 54, 56] (MSE is largely positive) while, on the other hand, when used in methods based on the FDE theory they show usually a tendency to produce too low interaction energies ( $\Delta E$ is negative in most cases). On the contrary, hybrid functionals are quite accurate for the calculation of the interaction energies of chargetransfer complexes and, at the same time, corresponding embedding calculations reproduce with good accuracy the parent supermolecular GKS calculations.

Concerning the error on embedding energies $(\Delta E)$, we note that the methods using hybrid $\mathrm{XC}$ functionals definitely outperform the ones employing semilocal XC approximations, yielding average deviations more than twice smaller. This improvement can be partially reconduced, in analogy to what shown in Refs. 45, 46, to a smaller overlap of the subsystem densities (i.e. to a smaller SIE) at the hybrid level, that leads to a reduction of the error on embedding energy. This effect is best recognized if we consider a set of systems where only one subsystem is varying, as for $\mathrm{NH}_{3}$ interacting with $\mathrm{Cl}_{2}$, $\mathrm{ClF}$, and $\mathrm{F}_{2}$ respectively. In this case the value of $\Delta E$ is increasing from 2.6 to about $7 \mathrm{mHa}$ when the $\mathrm{Cl}$ atoms are replaced by $\mathrm{F}$ atoms and this increase nicely correlates to the fact that the fluorine atom is affected by a larger self-interaction error than chlorine, so that it is plagued by a stronger overestimation of the diffuseness of the electron density at semilocal level of theory. However, in general it is not possible to find a trend valid for all the systems due to the different details of the interaction when both subsystems are changed.

In fact, despite the inclusion of exact exchange in the calculations may help to reduce the errors on embedding energies by lowering the effective overlap between the two subsystems, this is not the only effect determining the accuracy of the GKS-FDE energy calculations. In particular for hybrid methods still a subtle error compensation between kinetic, relaxation, and exchange contributions occurs. Thus, to shed light on the different aspects determining the embedding energy error we can recur to the embedding energy decomposition introduced in Ref. 45. In Tab. II we report the energy error contributions $\Delta T+\Delta D$ and $\Delta X^{S}$ for all the hybrid methods (BHLYP and $\mathrm{PBE} 0$ ) and system considered. For each energy error contribution we report the MSE, MAE and MARE. The contributions due to kinetic $(\Delta T)$ and relaxation $(\Delta D)$ effects are reported summed together because both terms yield very large values (100-200\% of the binding energies) and with opposite sign, so they only contribute to the total error through a strong error cancellation.

The analysis of the $\Delta T+\Delta D$ contributions shows that the use of the subsystem electron densities from hybrid calculations can provide a significant improvement of the semilocal embedding energies (the MAEs are 1.35 and 2.19 to be compared with 2.54 and 2.61), although the errors are still larger than the global ones reported in Tab. 1) (MAEs of 0.94 and 0.90 for BHLYP and PBE0, respectively). This improvement is determined exclusively from the reduced overlap displayed by the densities obtained from hybrid calculations, since here no exchange approximation is considered. To make a quantitative evaluation of the effect it is possible to use the semilocal differential relative error (SDRE) statistical indicator, defined as

$$
\mathrm{SDRE}=\frac{1}{N} \sum_{i=1}^{N} \frac{\left|\Delta T_{i}+\Delta D_{i}\right|-\left|\Delta E_{i}^{\mathrm{GGA}}\right|}{E_{b, i}^{r e f}}
$$

with the sum extend over all the $N$ systems reported in the table. A negative value of SDRE indicates that using subsystem densities from a given hybrid calculation improves on average the calculation of kinetic and relaxation energy contributions over the use of GGA densities. Using the SDRE indicator it is thus possible to compare energetic error terms with the same explicit level of approximation, i.e. the semilocal kinetic energy approximation and the density relaxation error [45]. Looking at the values of SDRE for the two hybrids considered in the table, it can be seen that in both cases these methods outperform the corresponding semilocal ones (negative values of SDRE). This result reflects the good quality of the electron densities computed with hybrid embedding methods (see next section) and evidences once more the importance of the density overlap in this context. In fact, 
TABLE I: Dispersion-corrected binding energies resulting from conventional supermolecular DFT $\left(E_{b}^{\mathrm{GKS}}\right)$ and embedding $\left(E_{b}^{\mathrm{emb}}\right)$ calculations for several test charge-transfer complexes. The differences of the binding energies with respect to the reference ones are also reported $\left(\Delta^{\mathrm{GKS}}=E_{b}^{\mathrm{GKS}}-E_{b}^{r e f}\right.$ and $\left.\Delta^{\mathrm{emb}}=E_{b}^{\mathrm{emb}}-E_{b}^{r e f}\right)$ as well as the error on the embedding energy $\left(\Delta E\right.$; see Eq. (8) ). The reference binding energies $\left(E_{b}^{r e f}\right)$ are reported in the second column. In all calculations we used the revAPBEk functional for the non-additive kinetic energies and a supermolecular def2-TZVPPD basis set. In the last lines we report the mean absolute error (MAE), the mean signed error (MSE), and the mean absolute relative error (MARE). All values are in mHa.

\begin{tabular}{|c|c|c|c|c|c|c|c|c|c|c|c|}
\hline System & $E_{b}^{\text {ref }}$ & $E_{b}^{E_{b}^{\text {GKS }}}$ & $E_{b}^{\mathrm{emb}}$ & 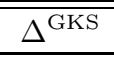 & $\Delta^{\mathrm{emb}}$ & $\Delta E$ & $E_{E_{b}^{\text {GKS }}}$ & $E_{b}^{\text {emb }}$ & 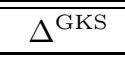 & $\Delta^{\mathrm{emb}}$ & $\Delta E$ \\
\hline & & \multicolumn{5}{|c|}{ BLYP } & \multicolumn{5}{|c|}{ BHLYP } \\
\hline $\mathrm{NF}_{3}-\mathrm{HCN}$ & $1.67^{a}$ & 0.96 & 1.40 & -0.71 & -0.27 & $\overline{0.44}$ & 1.71 & 1.11 & 0.04 & -0.56 & -0.60 \\
\hline $\mathrm{C}_{2} \mathrm{H}_{4}-\mathrm{F}_{2}$ & $1.69^{b}$ & 5.21 & 0.56 & 3.52 & -1.13 & -4.65 & 2.24 & 0.11 & 0.55 & -1.58 & -2.13 \\
\hline $\mathrm{NF}_{3}-\mathrm{HNC}$ & $2.31^{a}$ & 2.93 & 3.07 & 0.62 & 0.76 & 0.14 & 2.56 & 2.35 & 0.25 & 0.04 & -0.21 \\
\hline $\mathrm{C}_{2} \mathrm{H}_{4}-\mathrm{Cl}_{2}$ & $2.60^{c}$ & 6.25 & 4.76 & 3.65 & 2.16 & -1.49 & 3.81 & 3.91 & 1.21 & 1.31 & 0.10 \\
\hline $\mathrm{NH}_{3}-\mathrm{F}_{2}$ & $2.88^{b}$ & 8.22 & 1.16 & 5.34 & -1.72 & -7.06 & 3.51 & 0.99 & 0.63 & -1.89 & -2.52 \\
\hline $\mathrm{NF}_{3}-\mathrm{ClF}$ & $2.92^{a}$ & 4.14 & 2.14 & 1.22 & -0.78 & -2.00 & 2.89 & 1.40 & -0.03 & -1.52 & -1.49 \\
\hline $\mathrm{NF}_{3}-\mathrm{HF}$ & $2.92^{a}$ & 4.14 & 3.22 & 1.22 & 0.30 & -0.92 & 3.52 & 2.71 & 0.60 & -0.21 & -0.81 \\
\hline $\mathrm{C}_{2} \mathrm{H}_{2}-\mathrm{ClF}$ & $6.07^{b}$ & 8.74 & 5.09 & 2.67 & -0.98 & -3.65 & 6.96 & 5.26 & 0.89 & -0.81 & -1.70 \\
\hline $\mathrm{HCN}-\mathrm{ClF}$ & $7.74^{b}$ & 8.17 & 6.70 & 0.43 & -1.04 & -1.47 & 7.90 & 7.27 & 0.16 & -0.47 & -0.63 \\
\hline $\mathrm{NH}_{3}-\mathrm{Cl}_{2}$ & $7.78^{b}$ & 11.82 & 9.23 & 4.04 & 1.45 & -2.59 & 8.59 & 8.67 & 0.81 & 0.89 & 0.08 \\
\hline $\mathrm{H}_{2} \mathrm{O}-\mathrm{ClF}$ & $8.54^{b}$ & 10.30 & 8.11 & 1.76 & -0.43 & -2.19 & 9.60 & 8.77 & 1.06 & 0.23 & -0.83 \\
\hline $\mathrm{NH}_{3}-\mathrm{ClF}$ & $16.92^{b}$ & 25.13 & 21.29 & 8.21 & 4.37 & -3.84 & 21.03 & 20.84 & 4.11 & 3.92 & -0.19 \\
\hline MSE & & & & 2.66 & 0.22 & -2.44 & & & 0.86 & -0.05 & -0.91 \\
\hline MAE & & & & 2.78 & 1.28 & 2.54 & & & 0.86 & 1.12 & 0.94 \\
\hline \multirow[t]{2}{*}{ MARE } & & \multicolumn{5}{|c|}{$71.47 \% \quad 31.23 \% \quad 72.56 \%$} & \multicolumn{5}{|c|}{$16.63 \%$} \\
\hline & & \multicolumn{5}{|c|}{$\mathrm{PBE}$} & \multicolumn{5}{|c|}{ PBE0 } \\
\hline $\mathrm{NF}_{3}-\mathrm{HCN}$ & $1.67^{a}$ & 1.42 & 1.83 & -0.25 & 0.16 & $\overline{0.41}$ & 1.48 & 1.91 & -0.19 & 0.24 & 0.43 \\
\hline $\mathrm{C}_{2} \mathrm{H}_{4}-\mathrm{F}_{2}$ & $1.69^{b}$ & 5.39 & 1.11 & 3.70 & -0.58 & -4.28 & 3.05 & 1.13 & 1.36 & -0.56 & -1.92 \\
\hline $\mathrm{NF}_{3}-\mathrm{HNC}$ & $2.31^{a}$ & 3.49 & 3.61 & 1.18 & 1.30 & 0.12 & 2.97 & 3.48 & 0.66 & 1.17 & 0.51 \\
\hline $\mathrm{C}_{2} \mathrm{H}_{4}-\mathrm{Cl}_{2}$ & $2.60^{c}$ & 7.61 & 6.09 & 5.06 & 3.54 & -1.52 & 5.96 & 6.38 & 3.41 & 3.83 & 0.42 \\
\hline $\mathrm{NH}_{3}-\mathrm{F}_{2}$ & $2.88^{b}$ & 8.73 & 1.83 & 5.85 & -1.05 & -6.90 & 4.87 & 1.89 & 1.99 & -0.99 & -2.98 \\
\hline $\mathrm{NF}_{3}-\mathrm{ClF}$ & $2.92^{a}$ & 5.01 & 2.87 & 2.09 & -0.05 & -2.14 & 3.59 & 2.77 & 0.67 & -0.15 & -0.82 \\
\hline $\mathrm{NF}_{3}-\mathrm{HF}$ & $2.92^{a}$ & 4.73 & 3.82 & 1.81 & 0.90 & -0.91 & 4.02 & 3.80 & 1.10 & 0.88 & -0.22 \\
\hline $\mathrm{C}_{2} \mathrm{H}_{2}-\mathrm{ClF}$ & $6.07^{b}$ & 10.56 & 6.85 & 4.49 & 0.78 & -3.71 & 8.84 & 7.33 & 2.77 & 1.26 & -1.51 \\
\hline $\mathrm{HCN}-\mathrm{ClF}$ & $7.74^{b}$ & 9.63 & 8.01 & 1.89 & 0.27 & -1.62 & 8.46 & 8.43 & 0.72 & 0.69 & -0.03 \\
\hline $\mathrm{NH}_{3}-\mathrm{Cl}_{2}$ & $7.78^{b}$ & 13.48 & 10.64 & 5.70 & 2.86 & -2.84 & 10.90 & 10.69 & 3.12 & 2.91 & -0.21 \\
\hline $\mathrm{H}_{2} \mathrm{O}-\mathrm{ClF}$ & $8.54^{b}$ & 11.63 & 9.21 & 3.09 & 0.67 & -2.42 & 10.21 & 9.76 & 1.67 & 1.22 & -0.45 \\
\hline $\mathrm{NH}_{3}-\mathrm{ClF}$ & $16.92^{b}$ & 28.84 & 24.40 & 11.92 & 7.48 & -4.44 & 25.62 & 24.31 & 8.70 & 7.39 & -1.31 \\
\hline MSE & & & & 3.88 & 1.36 & -2.52 & & & 2.17 & 1.49 & -0.67 \\
\hline MAE & & & & 3.92 & 1.64 & 2.61 & & & 2.20 & 1.77 & 0.90 \\
\hline MARE & & & & $91.53 \%$ & $4.43 \%$ & $1.65 \%$ & & & $45.82 \%$ & $36.92 \%$ & $29.83 \%$ \\
\hline
\end{tabular}

${ }^{a} \operatorname{CCSD}(\mathrm{T})-\mathrm{F} 12 \mathrm{~b} / \mathrm{VTZ}-\mathrm{F} 12$, Ref. 49.

${ }^{b} \mathrm{~W} 1$ benchmark calculations, Ref. 74 .

${ }^{c} \operatorname{CCSD}(\mathrm{T})$ extrapolated to CBS limit, this work.

the larger improvement is obtained for BHLYP (SDRE of $-33.51 \%$ ) due to its higher content of exact exchange with respect to PBE0 (SDRE of $-12.97 \%$ ).

Considering the exchange contributions to the embedding error $\left(\Delta X^{S}\right)$ we note immediately that, for both methods, they are in general of opposite sign and of the same order of magnitude as the $\Delta T+\Delta D$ terms. Hence, an important error cancellation effect occurs in all cases. This effect can be measured by considering the exchange differential error (XDE) and the exchange differential rel- 
TABLE II: Kinetic-relaxation $(\Delta T+\Delta D)$, and exchange $\left(\Delta X^{S}\right)$ contributions to the embedding energy error (see Ref. 45 for details). In all calculations we used the revAPBEk functional for the non-additive kinetic energies and a supermolecular def2-TZVPPD basis set. At the bottom of each panel the mean signed error (MSE), mean absolute error (MAE) and the mean absolute relative error (MARE) are reported. For the $\Delta T+\Delta D$ values also the semilocal differential relative error (SDRE; see text) is reported. For $\Delta X^{S}$ we computed the exchange differential error (XDE) and the exchange differential relative error (XDRE) [45]. All values are in mHa.

\begin{tabular}{|c|c|c|}
\hline Systems & BHLYP & PBE0 \\
\hline & \multicolumn{2}{|c|}{$\Delta T+\Delta D$} \\
\hline $\mathrm{NF}_{3}-\mathrm{HCN}$ & -0.4 & -0.4 \\
\hline $\mathrm{C}_{2} \mathrm{H}_{4}-\mathrm{F}_{2}$ & 2.6 & 3.4 \\
\hline $\mathrm{NF}_{3}-\mathrm{HNC}$ & -0.3 & -0.2 \\
\hline $\mathrm{C}_{2} \mathrm{H}_{4}-\mathrm{Cl}_{2}$ & 0.3 & 1.1 \\
\hline $\mathrm{NH}_{3}-\mathrm{F}_{2}$ & 3.7 & 5.5 \\
\hline $\mathrm{NF}_{3}-\mathrm{ClF}$ & 1.1 & 1.8 \\
\hline $\mathrm{NF}_{3}-\mathrm{HF}$ & 0.6 & 0.8 \\
\hline $\mathrm{C}_{2} \mathrm{H}_{2}-\mathrm{ClF}$ & 2.2 & 3.3 \\
\hline $\mathrm{HCN}-\mathrm{ClF}$ & 0.4 & 1.2 \\
\hline $\mathrm{NH}_{3}-\mathrm{Cl}_{2}$ & 0.9 & 2.3 \\
\hline $\mathrm{H}_{2} \mathrm{O}-\mathrm{ClF}$ & 0.8 & 1.9 \\
\hline $\mathrm{NH}_{3}-\mathrm{ClF}$ & 2.9 & 4.3 \\
\hline$\overline{\mathrm{MAE}}$ & 1.35 & 2.19 \\
\hline MSE & 1.23 & 2.09 \\
\hline MARE & $39.19 \%$ & $58.65 \%$ \\
\hline \multirow[t]{2}{*}{ SDRE } & $-33.51 \%$ & $-12.97 \%$ \\
\hline & \multicolumn{2}{|c|}{$\Delta X^{S}$} \\
\hline $\mathrm{NF}_{3}-\mathrm{HCN}$ & 1.0 & -0.0 \\
\hline $\mathrm{C}_{2} \mathrm{H}_{4}-\mathrm{F}_{2}$ & -0.4 & -1.5 \\
\hline $\mathrm{NF}_{3}-\mathrm{HNC}$ & 0.5 & -0.3 \\
\hline $\mathrm{C}_{2} \mathrm{H}_{4}-\mathrm{Cl}_{2}$ & -0.4 & -1.6 \\
\hline $\mathrm{NH}_{3}-\mathrm{F}_{2}$ & -1.2 & -2.5 \\
\hline $\mathrm{NF}_{3}-\mathrm{ClF}$ & 0.4 & -0.9 \\
\hline $\mathrm{NF}_{3}-\mathrm{HF}$ & 0.2 & -0.6 \\
\hline $\mathrm{C}_{2} \mathrm{H}_{2}-\mathrm{ClF}$ & -0.5 & -1.7 \\
\hline $\mathrm{HCN}-\mathrm{ClF}$ & 0.3 & -1.2 \\
\hline $\mathrm{NH}_{3}-\mathrm{Cl}_{2}$ & -1.0 & -2.1 \\
\hline $\mathrm{H}_{2} \mathrm{O}-\mathrm{ClF}$ & 0.0 & -1.5 \\
\hline $\mathrm{NH}_{3}-\mathrm{ClF}$ & -2.7 & -3.0 \\
\hline MAE & 0.72 & 1.41 \\
\hline MSE & -0.32 & -1.41 \\
\hline MARE & $19.10 \%$ & $34.10 \%$ \\
\hline$\overline{\mathrm{XDE}}$ & -0.41 & -1.28 \\
\hline XDRE & $-6.79 \%$ & $-28.83 \%$ \\
\hline
\end{tabular}

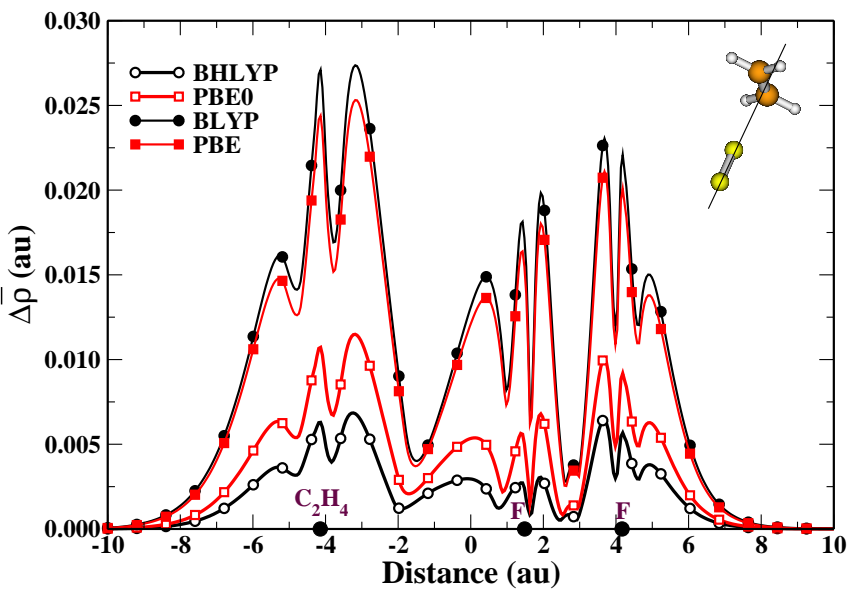

FIG. 1: Absolute deviation from the reference supermolecular electron density of plane-averaged embedding densities obtained from different embedding approaches, for the $\mathrm{C}_{2} \mathrm{H}_{4}$ $\mathrm{F}_{2}$ complex. The filled circles on the $x$-axis denote the atoms' positions.

ative error (XDRE) statistical indicators 45]

$$
\begin{aligned}
\mathrm{XDE} & =\frac{1}{N} \sum_{i=1}^{N}\left|\Delta E_{i}\right|-\left|\Delta T_{i}+\Delta D_{i}\right|, \\
\mathrm{XDRE} & =\frac{1}{N} \sum_{i=1}^{N} \frac{\left|\Delta E_{i}\right|-\left|\Delta T_{i}+\Delta D_{i}\right|}{E_{b, i}^{r e f}},
\end{aligned}
$$

which provide (by increasingly negative values) an estimation of the improvement due to the error cancellation effect induced by the additional approximation on the exchange nonadditive embedding terms. For BHLYP and PBE0 the XDE and XDRE values are negative, indicating, as expected, an error cancellation between the kinetic-relaxation and the exchange contributions. However, the XDE is three times larger for PBE0 than for BHLYP, so in the former case a much more effective error cancellation occurs. This explains why PBE0 has the smaller energy error $\Delta E$ in Tab. I, despite it has kineticrelaxation errors significantly larger than BHLYP.

\section{Embedding density}

In this section we consider the ability of different embedding methods to reproduce the electron density of supermolecular charge-transfer systems. This is an important test for embedding approaches and provides direct insight into the quality of the embedding potential [40, 41, 44, 46, 91 94]. To discuss this issue we report in Fig. 11 the plot of the absolute deviation of the planeaveraged densities $\left(\Delta \bar{\rho}\right.$; see Eq. (5) ) for the $\mathrm{C}_{2} \mathrm{H}_{4}-\mathrm{F}_{2}$ complex, as an example. For other systems similar plots are obtained (not reported). 
The figure shows that, unlike for hydrogen bond systems [44, 46], which are prototypical examples for FDEbased studies, the density errors are quite different for different methods, especially if hybrid and GGA approaches are compared. This trend can be well captured by considering the integrated errors on embedding densities $(\xi$; see Eq. (6) ), as resulting from different methods, which are reported in Tab. III] These data, along with the pro-

TABLE III: Valence density errors $\xi$ for GKS-FDE calculations (BHLYP and PBE0) and FDE-based calculations using conventional BLYP and PBE functionals. In all calculations we used the revAPBEk functional for the nonadditive kinetic energies and a supermolecular def2-TZVPPD basis set. In the last line the mean absolute error (MAE) is reported.

\begin{tabular}{lcccc}
\hline \hline & \multicolumn{2}{c}{ Hybrid } & \multicolumn{2}{c}{ Semilocal } \\
Systems & BHLYP & PBE0 & BLYP & PBE \\
\hline $\mathrm{NF}_{3}-\mathrm{HCN}$ & 0.13 & 0.24 & 0.29 & 0.29 \\
$\mathrm{C}_{2} \mathrm{H}_{4}-\mathrm{F}_{2}$ & 1.58 & 2.75 & 6.94 & 6.35 \\
$\mathrm{NF}_{3}-\mathrm{HNC}$ & 0.41 & 0.49 & 0.58 & 0.58 \\
$\mathrm{C}_{2} \mathrm{H}_{4}-\mathrm{Cl}_{2}$ & 3.34 & 4.31 & 5.83 & 5.77 \\
$\mathrm{NH}_{3}-\mathrm{F}_{2}$ & 2.24 & 4.36 & 9.84 & 9.59 \\
$\mathrm{NF}_{3}-\mathrm{ClF}$ & 0.64 & 0.99 & 1.64 & 1.73 \\
$\mathrm{NF}_{3}-\mathrm{HF}$ & 0.60 & 0.74 & 0.96 & 0.94 \\
$\mathrm{C}_{2} \mathrm{H}_{2}-\mathrm{ClF}$ & 3.14 & 4.32 & 6.03 & 6.02 \\
$\mathrm{HCN}_{-} \mathrm{ClF}$ & 1.76 & 2.33 & 3.13 & 3.21 \\
$\mathrm{NH}_{3}-\mathrm{Cl}$ & 3.98 & 5.48 & 7.45 & 7.60 \\
$\mathrm{H}_{2} \mathrm{O}-\mathrm{ClF}$ & 2.37 & 3.41 & 4.89 & 5.06 \\
$\mathrm{NH}_{3}-\mathrm{ClF}$ & 8.07 & 9.37 & 11.27 & 11.15 \\
& & & & \\
$\mathrm{MAE}$ & 2.35 & 3.23 & 4.90 & 4.86 \\
\hline \hline
\end{tabular}

portionality of the $\Delta \bar{\rho}(z)$ profiles reported in Fig. 1 (the embedding errors in the density have a similar spatial distribution), show that for all systems the errors on embedded densities are directly correlated to the amount of exact exchange included in the calculations (see also next section). In fact, the best results are found for BHLYP, which includes $50 \%$ of Hartree-Fock exchange and has a MAE of only 2.35, comparable to that found in the case of hydrogen-bond and dipole-dipole interactions [44, 46]. On the contrary, methods based on GGA XC functionals yield significantly larger errors, more than twice as large as the BHLYP ones. We note that such large variations of the embedding density error with the method are rather unusual and are not encountered in the case of hydrogen bond and dipole-dipole complexes [44, 46]. This indicates the special relevance of the inclusion of exact exchange for the treatment of charge-transfer complexes via FDEbased methods and fully agrees with the analysis made in the previous section about the role of relaxation effects for the energy errors of different methods.

Beside the total quality of the embedding density, in the present context another quantity of great interest is
TABLE IV: Charge transfer $\chi$ resulting from conventional supermolecular DFT and embedding calculations for several test charge-transfer complexes. The mean absolute error (MAE), the mean signed error (MSE), and the mean absolute relative error (MARE) respect to the reference MP2 charge transfer are also reported ${ }^{a}$.

\begin{tabular}{|c|c|c|c|c|c|}
\hline Systems & $\chi^{\mathrm{KS}}$ & $\chi^{\mathrm{emb}}$ & $\chi^{\mathrm{GKS}}$ & $\chi^{\mathrm{emb}}$ & MP2 \\
\hline & \multicolumn{2}{|c|}{ BLYP } & \multicolumn{2}{|c|}{ BHLYP } & \\
\hline $\mathrm{NF}_{3}-\mathrm{HCN}$ & 0.017 & 0.011 & 0.012 & 0.010 & 0.009 \\
\hline $\mathrm{C}_{2} \mathrm{H}_{4}-\mathrm{F}_{2}$ & 0.082 & 0.014 & 0.029 & 0.015 & 0.018 \\
\hline $\mathrm{NF}_{3}-\mathrm{HNC}$ & 0.007 & 0.007 & 0.006 & 0.006 & 0.005 \\
\hline $\mathrm{C}_{2} \mathrm{H}_{4}-\mathrm{Cl}_{2}$ & 0.093 & 0.044 & 0.061 & 0.045 & 0.053 \\
\hline $\mathrm{NH}_{3}-\mathrm{F}_{2}$ & 0.105 & 0.018 & 0.032 & 0.015 & 0.026 \\
\hline $\mathrm{NF}_{3}-\mathrm{ClF}$ & 0.035 & 0.013 & 0.015 & 0.009 & 0.013 \\
\hline $\mathrm{NF}_{3}-\mathrm{HF}$ & 0.025 & 0.013 & 0.018 & 0.012 & 0.018 \\
\hline $\mathrm{C}_{2} \mathrm{H}_{2}-\mathrm{ClF}$ & 0.102 & 0.051 & 0.067 & 0.049 & 0.052 \\
\hline $\mathrm{HCN}-\mathrm{ClF}$ & 0.050 & 0.028 & 0.033 & 0.025 & 0.034 \\
\hline $\mathrm{NH}_{3}-\mathrm{Cl}_{2}$ & 0.122 & 0.060 & 0.064 & 0.044 & 0.061 \\
\hline $\mathrm{H}_{2} \mathrm{O}-\mathrm{ClF}$ & 0.072 & 0.034 & 0.041 & 0.028 & 0.042 \\
\hline $\mathrm{NH}_{3}-\mathrm{ClF}$ & 0.217 & 0.209 & 0.163 & 0.126 & 0.177 \\
\hline MSE & 0.035 & -0.001 & 0.003 & -0.011 & \\
\hline MAE & 0.035 & 0.007 & 0.005 & 0.011 & \\
\hline \multirow[t]{2}{*}{ MARE } & $118.07 \%$ & $18.64 \%$ & $17.50 \%$ & $25.63 \%$ & \\
\hline & \multicolumn{2}{|c|}{ PBE } & \multicolumn{2}{|c|}{ PBE0 } & \\
\hline $\mathrm{NF}_{3}-\mathrm{HCN}$ & 0.016 & 0.011 & 0.013 & 0.011 & 0.009 \\
\hline $\mathrm{C}_{2} \mathrm{H}_{4}-\mathrm{F}_{2}$ & 0.074 & 0.013 & 0.039 & 0.013 & 0.018 \\
\hline $\mathrm{NF}_{3}-\mathrm{HNC}$ & 0.007 & 0.007 & 0.006 & 0.006 & 0.005 \\
\hline $\mathrm{C}_{2} \mathrm{H}_{4}-\mathrm{Cl}_{2}$ & 0.093 & 0.044 & 0.074 & 0.044 & 0.053 \\
\hline $\mathrm{NH}_{3}-\mathrm{F}_{2}$ & 0.101 & 0.017 & 0.052 & 0.015 & 0.026 \\
\hline $\mathrm{NF}_{3}-\mathrm{ClF}$ & 0.037 & 0.013 & 0.024 & 0.011 & 0.013 \\
\hline $\mathrm{NF}_{3}-\mathrm{HF}$ & 0.025 & 0.013 & 0.020 & 0.013 & 0.018 \\
\hline $\mathrm{C}_{2} \mathrm{H}_{2}-\mathrm{ClF}$ & 0.101 & 0.050 & 0.081 & 0.049 & 0.052 \\
\hline $\mathrm{HCN}-\mathrm{ClF}$ & 0.051 & 0.028 & 0.040 & 0.027 & 0.034 \\
\hline $\mathrm{NH}_{3}-\mathrm{Cl}_{2}$ & 0.125 & 0.061 & 0.089 & 0.050 & 0.061 \\
\hline $\mathrm{H}_{2} \mathrm{O}-\mathrm{ClF}$ & 0.074 & 0.035 & 0.054 & 0.030 & 0.042 \\
\hline $\mathrm{NH}_{3}-\mathrm{ClF}$ & 0.221 & 0.206 & 0.191 & 0.162 & 0.177 \\
\hline MSE & 0.035 & -0.001 & 0.015 & -0.006 & \\
\hline MAE & 0.035 & 0.006 & 0.015 & 0.007 & \\
\hline MARE & $113.80 \%$ & $19.26 \%$ & $48.22 \%$ & $21.00 \%$ & \\
\hline
\end{tabular}

${ }^{a}$ The statistical indicators are computed using the error $\Delta \chi^{\ell}=\chi^{\ell}-\chi^{\mathrm{MP} 2}$ with $\ell=\mathrm{KS}, \mathrm{GKS}, \mathrm{emb}$.

the net charge transfer predicted by different methods for the complexes under examination. The results for the various approaches, together with reference MP2 values are reported in Tab. IV]

The table shows the well-known tendency of the GGA XC functionals to overestimate the charge transfer 50, 51], so that BLYP and PBE yield for $\chi^{\text {GKS }}$ MAREs 
well above $100 \%$, while much better results (MARE of $17.5 \%)$ are obtained by including a large fraction of exact exchange, as in BHLYP. However, a different situation is observed when embedding calculations are considered. These have, in fact, a marked tendency towards an underestimation of the charge transfer, compared to the corresponding supermolecular calculation on the whole system, probably related to the excessive repulsive character of the approximated embedding potential. This behavior is also much more pronounced for GGA methods than for hybrids. As a consequence, the BHLYP results are slightly worsened compared to the reference MP2 ones when embedding is used, while the semilocal methods display a strong improvement.

In order to shine light on this finding, we consider that embedding densities computed at the semilocal level of theory, when used to compute the energy, provide higher energies for the complexes with respect to corresponding densities from supermolecular calculations (semilocal functionals stabilize the energy of charge-transfer complexes by overestimating the charge transfer to compensate for the absence of long-range interactions [49]). Because, on the other hand, the isolated systems are not affected by this problem, this leads in general to a reduction of the computed binding energy (see Tab. I), thus effectively compensating for the tendency of the semilocal $\mathrm{XC}$ functionals to over-bind. This explains the fact that in Tab. I. for semilocal functionals, $\Delta^{\mathrm{emb}}$ has a much smaller MAE and MARE than $\Delta^{\text {GKS }}$. It is worth noting that the same considerations also apply to the hybrid approaches, but in these cases the effect is smaller. In fact, PBE0 binding energies are slightly improved (on average) when computed via an embedding approach, while BHLYP results end up to suffer from a slight underbinding.

\section{Role of the amount of exact exchange}

In previous sections we evidenced the important role of the exact exchange for the description of embedding energies and densities, by comparing the performance of FDE-based methods using semilocal XC functionals with that of GKS-FDE approaches using hybrid functionals based on exactly the same semilocal approximation. To push this investigation one step further here we consider more generally the family of one-parameter hybrid functionals

$$
E_{\mathrm{xc}}^{\mathrm{hyb}}(\alpha)=\alpha E_{H F}+(1-\alpha) E_{\mathrm{x}}^{\mathrm{B} 88 \mathrm{x}}+E_{\mathrm{c}}^{\mathrm{LYP}}
$$

where $E_{H F}$ is the Hartree-Fock exchange, $\alpha$ is a parameter, and $E_{c}^{\mathrm{LYP}}$ is the Lee-Yang-Parr correlation energy functional [83]. Eq. (13) reduces to BLYP for $\alpha=0$, to BHLYP for $\alpha=0.5$, and was already used to study the role of exact exchange in embedding calculations of hydrogen-bond and dipole-dipole interacting systems in Ref. 45 .

Fig. 2 reports, as a function of $\alpha$, the errors on the embedding energies for selected systems as well as the individual contributions $\Delta T+\Delta D$ and $\Delta X^{S}$. For most of the systems the error $\Delta E$ is found to decrease with $\alpha$ until $\alpha \sim 0.5$, and then to slightly increase for larger values of the parameter. This behavior is a consequence of an error cancellation between the $\Delta T+\Delta D$ contribution (middle panel), which starts from a rather large positive value and decreases with $\alpha$, and the $\Delta X^{S}$ term (right panel), which shows instead an evident parabolic shape with minima located around $\alpha=0.3$ and provides mostly negative contributions for $\alpha<0.5$. This behavior is quite different from the one observed for dipole-dipole and hydrogen-bond interactions (see Figure I of Ref. 45), which is generally characterized by positive contributions of $\Delta X^{S}$, increasing with $\alpha$. The present finding thus shows that for charge-transfer systems a more delicate balancing must be found between the need to increase the exact-exchange contribution, in order to reduce the density overlap, and the necessity to obtain the correct description of long-range $\mathrm{XC}$ interactions in the hybrid functional.

The family of one-parameter hybrid functionals can be also be employed to study the effect of the exact exchange on the description of embedded densities. The result of this study was already partially anticipated in previous sections and it is clearly shown in Fig. 3. by increasing the amount of exact exchange the error on the embedding density is monotonically decreased. As mentioned in previous discussions and in Ref. 46 this behavior is mainly due to the reduction of the overlap of the subsystem densities consequent to the reduction of the SIE. Of course, the effect is somehow attenuated for very large values of the parameter $\alpha$ because of the increased importance of the semilocal approximation used for the exchange term in the GKS-FDE scheme in these cases, as shown in right panel of Fig. 2 .

Finally, we can consider the ability of methods including different amounts of exact exchange to correctly reproduce the charge transfer. We thus report in Fig. 4 the difference $\Delta \chi$ between the charge transfer computed for each value of $\alpha$, with or without the embedding treatment, and the reference values obtained from MP2 relaxed densities. The plot shows that considering full GKS calculations the charge transfer is generally overestimated at the GGA level and better agreement with the reference is only found when a significative amount of Hartree-Fock exchange is considered. On the other hand, for embedding calculations the GKS charge transfer can be only reproduced when exact exchange is included, while calculations using a semilocal XC potential yield a strong underestimation with respect to the corresponding supermolecular calculation. In fact, the charge transfer computed from embedding methods appears to 

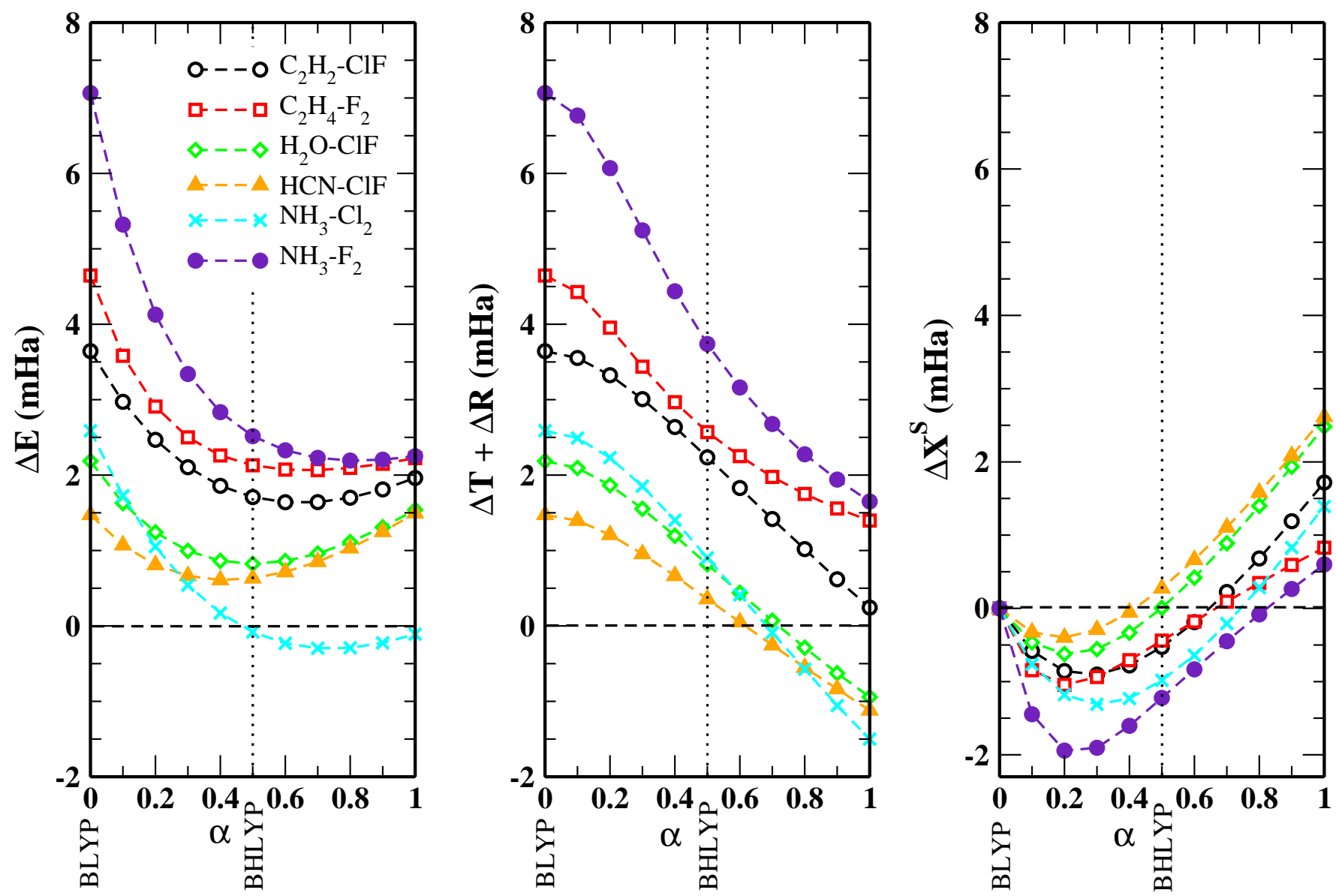

FIG. 2: Errors on the embedding energy for selected systems are reported in the left panel as computed with the family of functionals of Eq. (13). The corresponding contributions $\Delta T+\Delta D$ and $\Delta X^{S}$ are plotted in the middle and in the right panels, respectively. In all calculations we used the revAPBEk functional for the non-additive kinetic energies and a supermolecular def2-TZVPPD basis set.

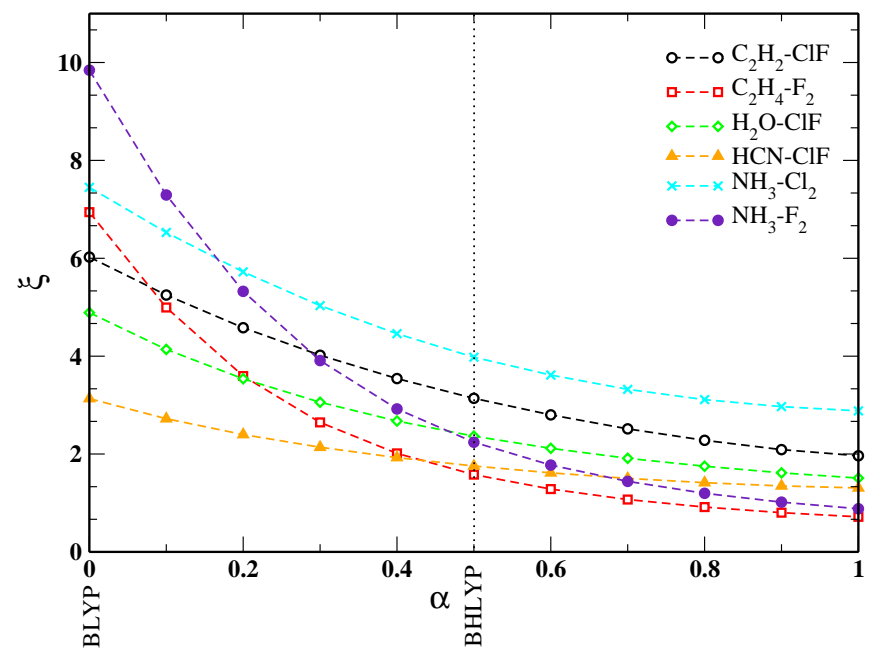

FIG. 3: Errors on the embedding density for selected systems as computed with the family of functionals of Eq. (13). In all calculations we used the revAPBEk functional for the nonadditive kinetic energies and a supermolecular def2-TZVPPD basis set. be almost independent from the percentage of exact exchange included in the $\mathrm{XC}$ functional. To understand these results we must consider that within FDE-based methods the number of electrons on each subsystem is fixed a priori, so that no real charge transfer between them can occur. The charge transfer must be instead mimicked by a strong polarization of the two subsystem densities, in such a way that their sum will give the same spatial distribution as the electron density of the full system. The role of the embedding potential is thus to provide a polarization of the subsystems, while each subsystem must respond properly to this perturbation. In the case of semilocal functionals they are well known to provide a poor description of polarization [95], thus a poor description of charge transfer must be expected. On the contrary, hybrid functionals have been shown to give a good response to polarization perturbations within the GKS-FDE scheme [4], thus they can provide a good reproduction of the charge transfer between the subsystems. 

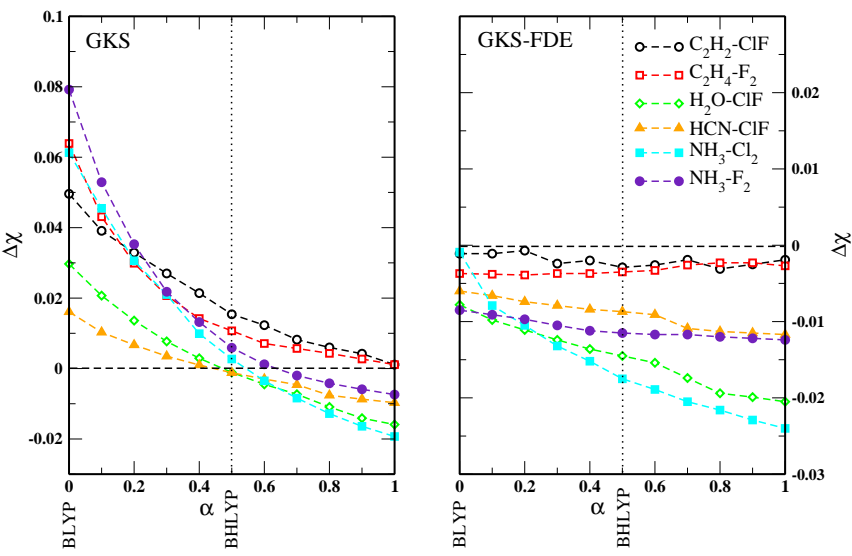

FIG. 4: Difference between the charge transfer computed with functionals defined by Eq. (13) and the reference MP2 value. In the left panel we report the results of GKS calculations on whole systems; in the right panel the corresponding embedding results are shown. In all calculations we used the revAPBEk functional for the non-additive kinetic energies and a supermolecular def2-TZVPPD basis set.

\section{Role of charge transfer}

In an analysis of the performance of embedding methods for the simulation of ground-state charge-transfer complexes it is obviously interesting to study in details what is the relationship between the outcome of the calculations and the magnitude of the charge transfer. However, this study cannot be performed straightforwardly by a simple comparison of different systems displaying different values of the charge transfer because in this way also many other details of the interaction are changed at the same time. For this reason in this section we prefer to focus the attention on a single system, namely the linear $\mathrm{HCN}-\mathrm{ClF}$ complex, and force a modulation of the charge transfer through the application of an external constant electric field directed along the axis of the complex.

In Fig. 5 we report the error on embedding energy $(\Delta E)$, the error on embedding density $(\xi)$, and the error on the computed net charge on the dihalogen monomer $\mathrm{ClF}\left(\nu^{\mathrm{emb}}-\nu^{\mathrm{GKS}}\right)$ as a function of the charge transfer induced (at the GKS level) by the applied external field for the two functionals BHLYP and BLYP. The corresponding electric field is reported in the inset of Fig. 5 . The figure shows that both functionals display the same behavior with the induced charge transfer, with all errors increasing as the charge transfer grows. In fact, the minima of the embedding errors are approximately located at zero charge transfer. The energy error decomposition (not reported) confirms that the increase of the energy error is essentially due to a large increase in the kinetic and relaxation contributions.

Moreover, the lowest panel of Fig. 5 shows, in agreement with our analysis of previous sections, that the in-

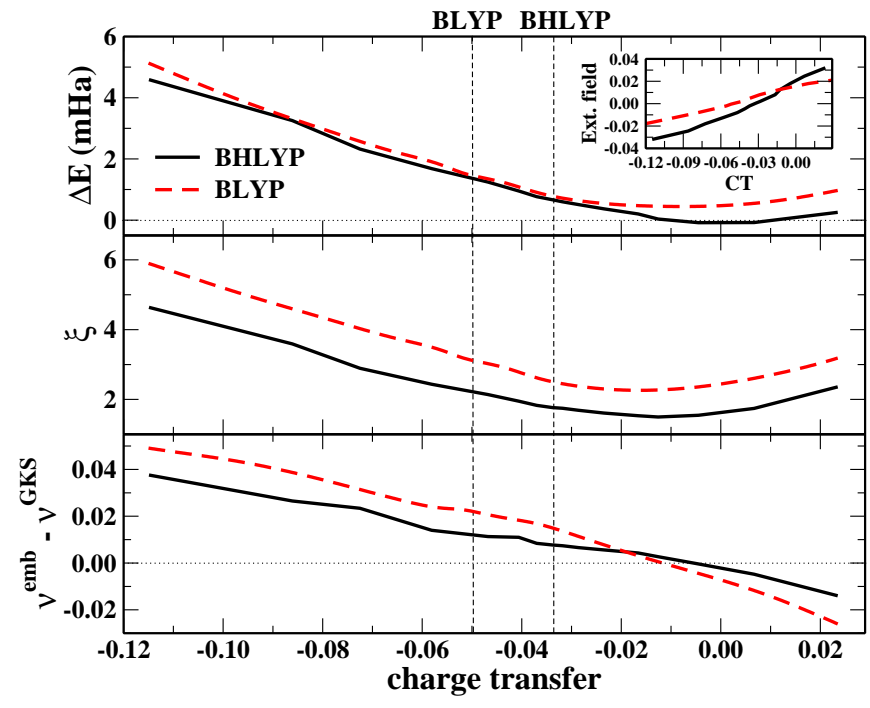

FIG. 5: Errors on embedding energy $(\Delta E)$, embedding density $(\xi)$, and the computed signed charge transfer on the dihalogen monomer $\mathrm{ClF}\left(\nu^{\mathrm{emb}}-\nu^{\mathrm{GKS}}\right)$ as a function of the charge transfer induced (at the GKS level) by the applied external field, for BHLYP and BLYP. The inset reports the external electric field used to generate different charge transfer values.

crease of charge transfer is in linear correspondence with an increase of the inaccuracy in its evaluation by embedding methods. This implies, as also shown by a comparison of the two upper panels of Fig. [5 with the lower one, that the embedding errors are correlated (almost linearly) with the inaccuracy of embedding approaches to correctly describe the amount of charge transfer.

\section{Role of geometry}

All previous investigations have been carried out at reference geometries. However, for charge-transfer complexes large differences in the equilibrium geometry can be expected for different methods. In particular, hybrid $\mathrm{XC}$ functionals are able to produce equilibrium structures very close to the reference ones but semilocal XC approximations lead to significantly shorter intermolecular distances [50 52, 54]. As shown in Ref. 45 the geometry and the intermolecular distance play an important role in determining the accuracy of embedding calculations. It is therefore interesting, in the present context, to assess this issue for the limits represented by the structures optimized at the semilocal BLYP and hybrid BHLYP level.

To this end we consider in Fig. 6 the error on embedding energy $(\Delta E)$, the error on embedding density $(\xi)$, and the error on the computed monomer charge $\left(\nu^{\mathrm{emb}}-\nu^{\mathrm{GKS}}\right)$ at different geometries interpolating linearly between the BLYP and the BHLYP optimized geometries of the complex $\mathrm{C}_{2} \mathrm{H}_{4}-\mathrm{F}_{2}$ (similar results are 


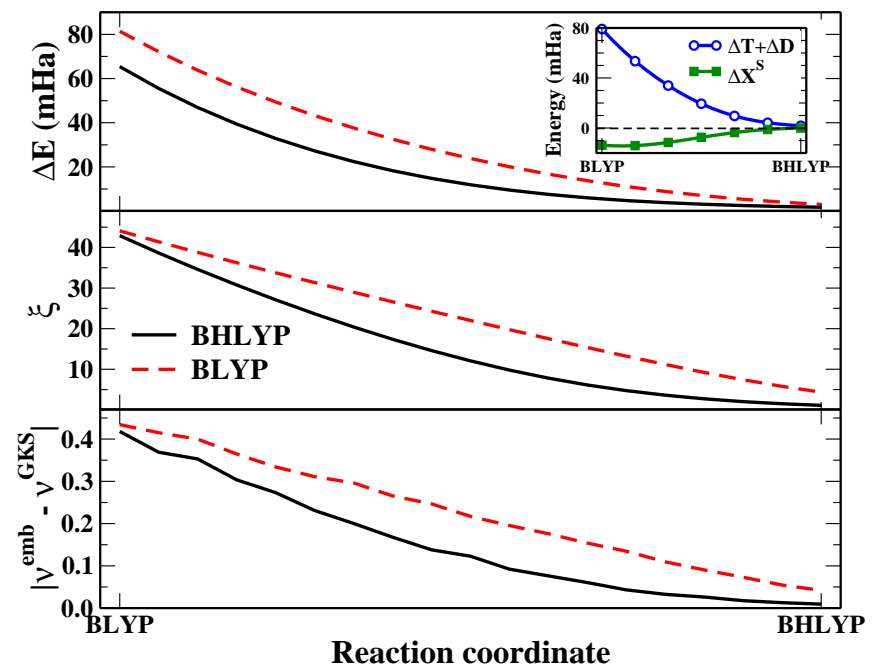

FIG. 6: Errors on embedding energy $(\Delta E)$, embedding density $(\xi)$, and the computed net charge on the ethylene monomer $\left(\nu^{\mathrm{emb}}-\nu^{\mathrm{GKS}}\right)$ at different geometries interpolating between the BLYP and BHLYP equilibrium geometry of the $\mathrm{C}_{2} \mathrm{H}_{4}-\mathrm{F}_{2}$ complex. In the inset the embedding error decomposition for the BHLYP case is reported.

found for other systems). The intermolecular distance between the $\mathrm{F}_{2}$ monomer and the plane of the ethylene is $1.91 \AA$ and $2.92 \AA$ for BLYP and BHLYP, respectively. The figure shows the important role of the geometry for all the embedding results. This finding can be understood considering that at small intermolecular distances, i.e. at the BLYP geometry, the overlap between the electron densities of the two subsystems rapidly grows. Moreover, because at reduced distances between the subsystems the orbital interactions are enhanced, also the charge transfer is highly increased, together with the error in its evaluation. As a consequence, large errors can be expected for the embedded densities, due to the wellknown limitations of nonadditive kinetic potentials for strongly overlapping densities [25, 45]. Similarly, large errors are found for the embedding energies. In this case an energy decomposition of the BHLYP embedding error (see inset of Fig. 6) further confirms that the increase of the error is essentially due to a large increase of the relaxation-kinetic term, while only a minor role is played by the exchange term $\Delta X^{S}$. Nevertheless, the latter being always opposite in sign to the relaxation-kinetic contribution provides a moderate error cancellation which improves the final performance of the hybrid method.

The results of Fig. 6 indicate in conclusion that, for the simulation of charge-transfer complexes via embedding approaches, the semilocal methods may display serious problems related to the use of relaxed structures (e.g. in geometry optimizations or molecular dynamics), beside the limitations already highlighted in the previous sections. Therefore, the hybrid approaches appear definitely more robust in such applications.

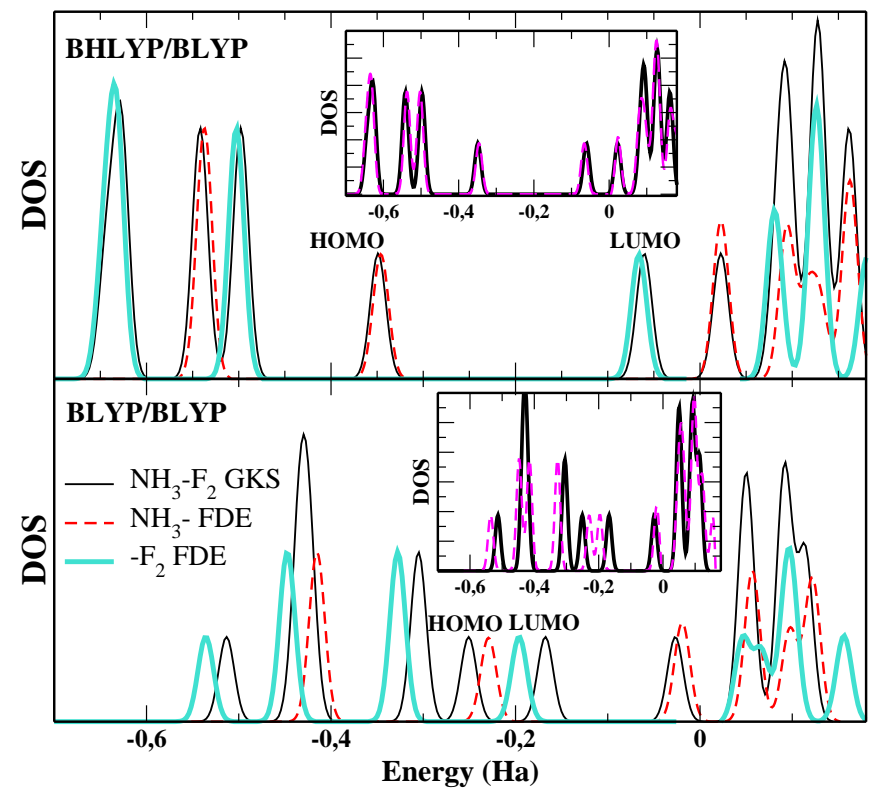

FIG. 7: Density of states (DOS) for both GKS and hybrid BHLYP (upper panel) and the semilocal BLYP (bottom panel) embedding calculations on the charge-transfer complex $\mathrm{NH}_{3}-$ $\mathrm{F}_{2}$. The solid black line indicates GKS DOS of the whole system; the red dashed and solid turquoise lines indicate DOS from embedding calculations on the monomers $\mathrm{NH}_{3}$ and $\mathrm{F}_{2}$ respectively. In the insets the GKS DOS (thick black line) is compared with the sum of the two subsystems DOSs (dashed magenta line). Energy values are in Ha.

\section{Orbital energies}

In previous sections we showed that only embedding approaches making use of hybrid XC functionals can reproduce the ground-state charge transfer of the investigated complexes with reasonable accuracy, while a strong underestimation occurs when semilocal approaches are used. This fact has important consequences on the accuracy of embedding calculations, that nevertheless are partially, or even mostly, mitigated by error compensation effects. The improper description of the charge transfer can have however more subtle effects, especially on the one-electron spectrum of the subsystems. This is an important issue, since the molecular orbitals of the embedded subsystems can be used as the basis for an analysis of the properties of the interacting subsystems and/or as input quantities in response calculations [96, 97].

The driving force for ground-state charge transfer in molecular complexes is a sizable difference in the chemical potential between the constituting (isolated) subsystems, so that, when the subsystems start to interact, a fraction of the electron density must be moved from one fragment to another to equilibrate it. The final chemical potential of the complex is an average between those of the initial non-interacting subsystems and it is determining the offset for the Kohn-Sham potential, thus de facto 
fixing the energy of the molecular orbitals. In an ideal exact FDE-based embedding calculation the embedding potential shall be able, by definition, to yield for the embedded subsystems the same chemical potential as for the full complex and, of course, shall induce a polarization able to mimic the exact charge-transfer. As a side consequence, the single-particle spectrum of the subsystems will be also correct (as compared to the corresponding supermolecular calculation). Similar conclusions apply for any approximated embedding calculation having sufficient accuracy. On the other hand, if the embedding procedure fails to reproduce the correct charge transfer (i.e. correct with respect to the calculation on the full system), the polarization exerted by the embedding potential on the two subsystems will be insufficient, thus the chemical potential of the two fragments will be rather different from the one of the full system (too large in one subsystem and too low in the other). Consequently, the molecular orbitals of the two subsystems will lay at too high (for one subsystem) and too low energies (for the other).

The two cases described above fit very well to the embedding calculations based on hybrid and semilocal functionals, respectively. This is nicely shown in Fig. 7 where, as an example, we report the density of states (DOS) of the $\mathrm{NH}_{3}-\mathrm{F}_{2}$ complex compared to that of the two embedded subsystems as resulting from BHLYP and BLYP embedding calculations. The comparison is in this case rather fair, since the two fragments composing the $\mathrm{NH}_{3}-\mathrm{F}_{2}$ complex interact only weakly so that the molecular orbitals of the full system can be easily attributed to either of the constituent subsystems. The figure shows very clearly that BHLYP embedding calculations yield subsystems orbitals which match well the ones from the full system (within $0.01 \mathrm{eV}$ ). Instead, the orbitals from the BLYP embedding calculations are systematically shifted with respect to the ones of the corresponding supermolecular calculation, with $\mathrm{F}_{2}$ orbitals shifted at lower energies and $\mathrm{NH}_{3}$ ones shifted at higher energies, consistently with the underestimation of charge transfer from $\mathrm{NH}_{3}$ to $\mathrm{F}_{2}$. Finally, we recall in favor of hybrid calculations that even supermolecular calculations using semilocal XC functionals provide a poor description of the single-particle spectrum, while better results can be only obtained by reducing the SIE $[98,99]$.

\section{CONCLUSIONS}

We applied methods based on the frozen density embedding theory to study the electronic properties of ground-state charge-transfer complexes and to clarify the ability of such approaches to treat accurately this class of systems and interactions. This topic is of particular interest due to the growing popularity of embedding approaches in the field of computational chemistry and the importance of charge-transfer complexes in many chemical applications. However, to date, embedding calculations have been mainly presented for hydrogen-bond and dipole-dipole interactions, while only few applications dealt with systems where charge transfer plays a significant role [36, 41, 68, 69].

Our work clarifies some of the motivations for this lack of embedding calculations concerning charge-transfer systems, showing that standard embedding approaches, making use of semilocal XC functionals, display several drawbacks in this context. Moreover, despite they can generally yield interaction energies of good quality (with respect to accurate references), this is due to a strong error cancellation effect and does not reflect a property of the corresponding Kohn-Sham supermolecular calculation. For these reasons and because semilocal XC functionals are known to perform rather poorly for chargetransfer complexes even in conventional Kohn-Sham supermolecular calculations, the use of such approaches does not appear appropriate.

The introduction of the GKS-FDE method mitigates different problems in the FDE-based calculations and thus provides a valuable tool to handle embedding calculations of charge-transfer systems. In fact, in the present work we showed the relevance of exact exchange contributions to reduce the embedding errors and improve the overall performance of the calculations, through a reduction of the Coulomb self-interaction error and a better description of subsystems' electronic properties.

In conclusion, FDE-based approaches making use of hybrid XC functionals appear as reliable and efficient methods to handle electronic structure calculations of systems characterized by charge-transfer interactions. This suggest the possibility of future studies in this direction making use of these tools to investigate chargetransfer interactions in complex environments.

This work was partially funded by the European Research Council (ERC) Starting Grant FP7 Project DEDOM, Grant No. 207441. The authors thank TURBOMOLE GmbH for providing the TURBOMOLE program package and M. Margarito for technical support.

[1] W. Yang, Phys. Rev. Lett. 66, 1438 (1991).

[2] W. Yang and T.-S. Lee, J. Chem. Phys. 103, 5674 (1995).

[3] T. Amaka, M. Kobayashi, and H. Nakai, J. Comput. Chem. 28, 2003 (2007).

[4] J. He, C. D. Paola, and L. Kantorovich, J. Chem. Phys. 130, 144104 (2009).

[5] L.-W. Wang, Z. Zhao, and J. Meza, Phys. Rev. B 77, 165113 (2008).

[6] Z. Zhao, J. Meza, and L.-W. Wang, J. Phys.: Cond. Matt. 20, 294203 (2008).

[7] S. Sugiki, N. Kurita, Y. Sengoku, and H. Sekino, Chem. Phys. Lett. 382, 611 (2003). 
[8] D. Fedorov and K. Kitaura, Chem. Phys. Lett. 389, 129 (2004).

[9] G. Senatore and K. R. Subbaswamy, Phys. Rev. B 34, 5754 (1986).

[10] P. Cortona, Phys. Rev. B 44, 8454 (1991).

[11] P. Cortona and A. Monteleone, Int. J. Quant. Chem. 52, 987 (1994).

[12] M. H. Cohen and A. Wasserman, J. Chem. Phys. A 111, 2229 (2007).

[13] M. H. Cohen, A. Wasserman, and K. Burke, J. Chem. Phys. A 111, 12447 (2007).

[14] P. Huang and E. A. Carter, J. Chem. Phys. 125, 084102 (2006).

[15] C. Huang and E. A. Carter, J. Chem. Phys. 135, 194104 (2011).

[16] M. H. Cohen, A. Wasserman, R. Car, and K. Burke, J. Chem. Phys. A 113, 2183 (2009).

[17] P. Elliott, M. H. Cohen, A. Wasserman, and K. Burke, J. Chem. Theory Comput. 5, 827 (2009).

[18] J. Nafziger, Q. Wu, and A. Wasserman, J. Chem. Phys. 135, 234101 (2011).

[19] M. S. Gordon, D. G. Fedorov, S. R. Pruitt, and L. V. Slipchenko, Chem. Rev. 112, 632 (2012).

[20] A. S. P. Gomes and C. R. Jacob, Annu. Rep. Prog. Chem., Sect. C: Phys. Chem. 108, 222 (2012).

[21] A. Solovyeva, M. Pavanello, and J. Neugebauer, J. Chem. Phys. 136, 194104 (2012).

[22] P. Hohenberg and W. Kohn, Phys. Rev. 136, B864 (1964).

[23] W. Kohn and L. J. Sham, Phys. Rev. 140, A1133 (1965).

[24] T. A. Wesolowski and A. Warshel, J. Phys. Chem. 97, 8050 (1993).

[25] T. A. Wesolowski, in Chemistry: Reviews of Current Trends, edited by J. Leszczynski (World Scientific, Singapore, 2006), vol. 10, p. 1.

[26] M. Dulak and T. A. Wesolowski, J. Mol. Model. 13, 631 (2007).

[27] R. Kevorkyants, M. Dulak, and T. A. Wesolowski, J. Chem. Phys. 124, 024104 (2006).

[28] T. A. Wesolowski, J. Chem. Phys. 106, 8516 (1997).

[29] T. A. Wesolowski, N. Vulliermet, and J. Weber, J. Mol. Struct. THEOCHEM 458, 151 (1998).

[30] F. Tran, J. Weber, T. A. Wesolowski, F. Cheikh, Y. Ellinger, and F. Pauzat, J. Phys. Chem. B 106, 8689 (2002).

[31] J. Neugebauer, M. J. Louwerse, E. J. Baerends, and T. A. Wesolowski, J. Chem. Phys. 122, 094115 (2005).

[32] C. R. Jacob, J. Neugebauer, and L. Visscher, J. Comput. Chem. 29, 1011 (2008).

[33] J. Neugebauer, C. R. Jacob, T. A. Wesolowski, and E. J. Baerends, J. Phys. Chem. A 109, 7805 (2005).

[34] C. R. Jacob, J. Neugebauer, L. Jensen, and L. Vissher, Phys. Chem. Chem. Phys. 8, 2349 (2006).

[35] F. R. Manby, M. Stella, J. D. Goodpaster, and T. F. Miller III, J. Chem. Theory Comput. 8, 2564 (2012).

[36] M. Pavanello and J. Neugebauer, J. Chem. Phys. 135, 234103 (2011).

[37] T. A. Wesolowski and A. Warshel, Chem. Phys. Lett. 248, 71 (1996).

[38] A. Lembarki and H. Chermette, Phys. Rev. A 50, 5328 (1994).

[39] L. A. Constantin, E. Fabiano, S. Laricchia, and F. Della Sala, Phys. Rev. Lett. 106, 186406 (2011).

[40] S. Laricchia, E. Fabiano, L. A. Constantin, and F. Della
Sala, J. Chem. Theory and Comput. 7, 2439 (2011).

[41] S. M. Beyhan, A. W. Götz, C. R. Jacob, and L. Visscher, J. Chem. Phys. 132, 044114 (2010).

[42] Y. Zhao and D. G. Truhlar, J. Chem. Theory Comput. 1, 415 (2005).

[43] E. Fabiano, L. A. Constantin, and F. D. Sala, J. Chem. Theory Comput. 7, 3548 (2011).

[44] S. Laricchia, E. Fabiano, and F. Della Sala, J. Chem. Phys. 133, 164111 (2010).

[45] S. Laricchia, E. Fabiano, and F. D. Sala, J. Chem. Phys. 137, 014102 (2012).

[46] S. Laricchia, E. Fabiano, and F. Della Sala, Chem. Phys. Lett. 518, 114 (2011).

[47] A. J. Cohen, P. Mori-Sánchez, and W. Yang, Science 321, 792 (2008).

[48] F. Della Sala, Theor. Chem. Acc. 117, 981 (2007).

[49] S. N. Steinmann, C. Piemontesi, A. Delachat, and C. Corminboeuf, J. Chem. Theory Comput. 8, 1629 (2012).

[50] E. Ruiz, D. R. Salahub, and A. Vela, J. Am. Chem. Soc. 117, 1141 (1995).

[51] E. Ruiz, D. R. Salahub, and A. Vela, J. Phys. Chem. 100, 12265 (1996).

[52] A. Garcia, E. M. Cruz, C. Sarasola, and J. M. Ugalde, J. Phys. Chem. A 101, 3021 (1997).

[53] A. Garcia, J. M. Elorza, and J. M. Ugalde, Journal of Molecular Structure: THEOCHEM 501 - 502, 207 (2000).

[54] A. Karpfen, Theor. Chem. Acc. 110, 1 (2003).

[55] M.-S. Liao, Y. Lu, V. D. Parker, and S. Scheiner, J. Phys. Chem. A 107, 8939 (2003).

[56] M.-S. Liao, Y. Lu, and S. Scheiner, J. Comput. Chem. 24, 623 (2003).

[57] E. L. Smith, D. Sadowsky, J. A. Phillips, C. J. Cramer, and D. J. Giesen, J. Phys. Chem. A 114, 2628 (2010).

[58] A. Y. Timoshkin and H. F. Schaefer III, J. Phys. Chem. C 112, 13816 (2008).

[59] J. A. Phillips and C. J. Cramer, J. Phys. Chem. B 111, 1408 (2007).

[60] B. Szefczyk, W. A. Sokalski, and J. Leszczynski, J. Chem. Phys. 117, 6952 (2002).

[61] X.-Y. Li and C.-X. Hu, J. Comput. Chem. 23, 874 (2002).

[62] T. Stein, L. Kronik, and R. Baer, J. Chem. Phys. 131, 244119 (2009).

[63] T. Manna and S. Bhattacharya, J, Mol. Model. 15, 885 (2009).

[64] S. Bhattacharya, Chem. Phys. Lett. 446, 199 (2007).

[65] C. López, R. M. Claramunt, E. Pinilla, M. R. Torres, I. Alkorta, and J. Elguero, Magn. Reson. Chem. 47, 917 (2009).

[66] E. Fabiano, M. Piacenza, S. D'Agostino, and F. Della Sala, J. Chem. Phys 131, 234101 (2009).

[67] F. Della Sala, E. Fabiano, S. Laricchia, S. D'Agostino, and M. Piacenza, Int. J. Quantum. Chem. 110, 2162 (2010).

[68] A. W. Götz, S. M. Beyhan, and L. Visscher, J. Chem. Theory Comput. 5, 3161 (2009).

[69] M. Dulak and T. A. Wesolowski, J. Chem. Phys. 124, 164101 (2006).

[70] J.-M. G. Lastra, J. W. Kaminski, and T. A. Wesolowski, J. Chem. Phys. 129, 074107 (2008).

[71] TURBOMOLE V6.2 2009, a development of University of Karlsruhe and Forschungszentrum Karlsruhe GmbH, 1989-2007, TURBOMOLE GmbH, since 200\%; available 
from http://www.turbomole.com.

[72] F. Weigend, F. Furche, and R. Ahlrichs, J. Chem. Phys. 119, 12753 (2003).

[73] F. Weigend and R. Ahlrichs, Phys. Chem. Chem. Phys. 7, 3297 (2005).

[74] Y. Zhao and D. G. Truhlar, J. Phys. Chem. 109, 5656 (2005).

[75] C. Møller and M. S. Plesset, Phys. Rev. 46, 618 (1934).

[76] T. H. Dunning, J. Chem. Phys. 90, 1007 (1989).

[77] R. A. Kendall, T. H. Dunning, and R. J. Harrison, J. Chem. Phys. 96, 6796 (1992).

[78] J. E. D. Bene, J. Phys. Chem. 97, 107 (1993).

[79] M. Piton̆ák, K. E. Riley, P. Neogrády, and P. Hobza, ChemPhysChem 9, 1636 (2008).

[80] K. Raghavachari, G. W. Trucks, J. A. Pople, and M. Head-Gordon, Chem. Phys. Lett. 157, 479 (1989).

[81] A. Halkier, T. Helgaker, P. Jørgensen, W. Klopper, H. Koch, J. Olsen, and A. K. Wilson, Chem. Phys. Lett. 286, 243 (1998).

[82] A. D. Becke, Phys. Rev. A 38, 3098 (1988).

[83] C. Lee, W. Yang, and R. G. Parr, Phys. Rev. B 37, 785 (1988).

[84] J. P. Perdew, K. Burke, and M. Ernzerhof, Phys. Rev. Lett. 77, 3865 (1996).

[85] J. P. Perdew, M. Ernzerhof, and K. Burke, J. Chem. Phys. 105, 9982 (1996).

[86] S. Grimme, J. Antony, S. Ehrlich, and H. Krieg, J. Chem. Phys. 132, 154104 (2010).
[87] J. I. Rodriguez, A. M. Köster, P. W. Ayers, A. SantosValle, A. Vela, and G. J. Merino, J. Comput. Chem. 30, 1082 (2009).

[88] S. F. Boys and F. Bernardi, Mol. Phys. 19, 553 (1970).

[89] J. Prissette, G. Seger, and E. Kochanski, J. Am. Chem. Soc. 100, 6941 (1978).

[90] T. A. Wesolowski, P.-Y. Morgantini, and J. Weber, J. Chem. Phys. 116, 6411 (2002).

[91] C. R. Jacob and L. Visscher, J. Chem. Phys. 128, 155102 (2008).

[92] K. Kiewisch, G. Eickerling, M. Reiher, and J. Neugebauer, J. Chem. Phys. 128, 044114 (2008).

[93] N. Govind, P. Sushko, W. Hess, M. Valiev, and K. Kowalski, Chem. Phys. Lett. 470, 353 (2009).

[94] S. Fux, C. R. Jacob, J. Neugebauer, L. Visscher, and M. Reiher, J. Chem. Phys. 132, 164101 (pages 18) (2010).

[95] Y. Zhao, N. E. Schultz, and D. G. Truhlar, J. Chem. Theory Comput. 2, 364 (2006).

[96] M. E. Casida and T. A. Wesolowski, Int. J. Quantum. Chem. 96, 577 (2004).

[97] J. Neugebauer, J. Chem. Phys. 126, 134116 (2007).

[98] F. Della Sala and A. Görling, J. Chem. Phys. 115, 5718 (2001).

[99] E. Fabiano and F. Della Sala, J. Chem. Phys. 126, 214102 (2007). 